\title{
Pleurography (thoracography) for pleural fistulas: A case series
}

\author{
Kenichi Watanabe, MD, PhD, Nobuhiro Imamura, MD, Jotaro Yusa, MD, Satoshi Koezuka, MD, and \\ Sumitaka Yamanaka, MD, PhD
}

\begin{abstract}
Background: Pleurography (PG) has been described previously but has not gained popularity. PG can determine the exact air leak points in the lung, which is important for treating pneumothorax and pleural fistulas. We believe that the usefulness of PG should be reassessed, and here we describe the method, air leak detection rate, and common complications.
\end{abstract}

Methods: From the 1210 cases of pleural fistulas that were treated at our institution between March 2015 and October 2018, 275 patients with recurrent primary pneumothorax or secondary spontaneous pneumothorax were selected for this study. PG was performed in 127 patients with persistent air leakage during exhalation. In addition, 35 patients with postoperative complications of air leakage persisting for 7 days or longer were included.

Results: Air leak points were detected in 119 patients (73\%), in the apex of the lung in 65 cases, in the basal segment in 13 cases, and in the middle lobe or lingular segment in 9 cases. There were 8 cases of hilar lesions, 12 cases of S6 lesions, 8 cases of upper lobe lesions other than apex, and 4 cases of upper mediastinal lesions. Complications within 30 days were observed in 10 cases $(6.2 \%)$, with 8 grade 2 cases involving fever, 1 grade 3 case involving infection, and one grade 1 case with abdominal distension.

Conclusions: The incidence of grade $\geq 3$ adverse events after PG was $0.6 \%$, which is considered acceptable. Our findings suggest that PG is a safe examination method to identify air leaks before surgery for pleural fistulas. (JTCVS Techniques 2021;7:285-91)

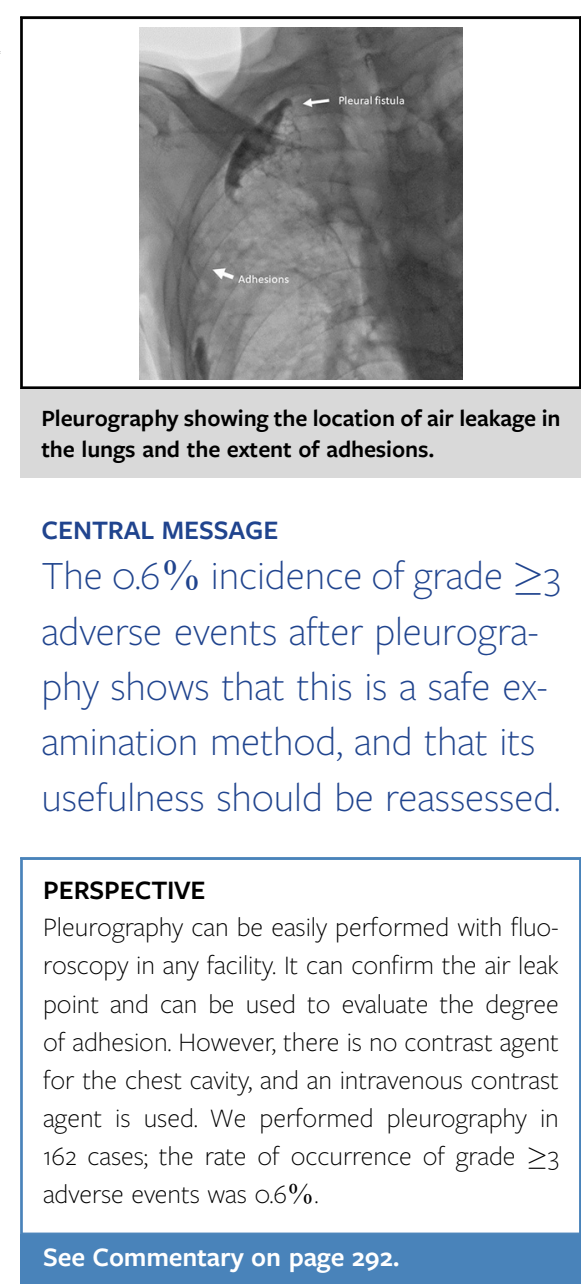

CENTRAL MESSAGE

The $0.6 \%$ incidence of grade $\geq 3$ adverse events after pleurography shows that this is a safe examination method, and that its usefulness should be reassessed.

\section{PERSPECTIVE}

Pleurography can be easily performed with fluoroscopy in any facility. It can confirm the air leak point and can be used to evaluate the degree of adhesion. However, there is no contrast agent for the chest cavity, and an intravenous contrast agent is used. We performed pleurography in 162 cases; the rate of occurrence of grade $\geq 3$ See Commentary on page 292.
- Video clip is available online.

From the Department of Thoracic Surgery, Pneumothorax Research Center, Nissan Tamagawa Hospital, Tokyo, Japan.

Received for publication March 12, 2021; accepted for publication March 16, 2021; available ahead of print March 23, 2021.

Address for reprints: Kenichi Watanabe, MD, PhD, Department of Thoracic Surgery, Pneumothorax Research Center, Nissan Tamagawa Hospital, 4-8-1, Seta Setagayaku, Tokyo 158-0095, Japan (E-mail: w-ken1@biglobe.jp).

2666-2507

Copyright (C) 2021 The Author(s). Published by Elsevier Inc. on behalf of The American Association for Thoracic Surgery. This is an open access article under the CC BY-NC-ND license (http://creativecommons.org/licenses/by-nc-nd/4.0/).

https://doi.org/10.1016/j.xjtc.2021.03.017
Pleurography (PG; also known as thoracography) was first reported in 1944 by Gordon and associates. ${ }^{1}$ Rudy and colleagues $^{2}$ reported its application in animals in 1968, and Hitomi and colleagues ${ }^{1}$ reported the PG method in 1971. Using a fluoroscopy table, 40 to $80 \mathrm{~mL}$ of meglumine iotalamate (Conley 60; Daiichi Sankyo, Tokyo, Japan) was injected into the pleural cavity, and the patient was repositioned to observe the pleural cavity.

Initially, the purpose of PG was to detect pleural tumors or mediastinal lesions. However, with the advent of computed tomography (CT), PG was no longer sought after for diagnosing pleural lesions, such as lung cancer and mediastinal tumors.

Takeno reported that PG was useful for the evaluation of pleural fistulas. ${ }^{3}$ The utility of PG has been recognized more 


\section{Abbreviations and Acronyms \\ BHDS $=$ Birt-Hogg-Dubé syndrome \\ $\mathrm{COPD}=$ chronic obstructive pulmonary disease \\ $\mathrm{CT}=$ Computed tomography \\ LAM $=$ lymphangioleiomyomatosis \\ $\mathrm{PG}=$ pleurography \\ PSP $=$ primary spontaneous pneumothorax \\ SSP = secondary spontaneous pneumothorax \\ $\mathrm{TEP}=$ thoracic endometoriotic pneumothorax}

recently, and it is now conventionally performed at several institutions in Japan. ${ }^{4}$ It has been argued that pleurodesis is superior to bullectomy for treating spontaneous pneumothorax ${ }^{5}$; however, some patients will require surgery. It has been reported that the frequency of occurrence of pleural fistulas after lobectomy is $58 \%$, and some cases require redo surgery. ${ }^{6}$ A method of detecting pleural fistulas with 3-dimensional cine CT was recently reported. ${ }^{7}$ Locating the air leak points in the lung is important in the treatment of pneumothorax and pleural fistulas so that an incision can be made at the most suitable location to shorten the operation time and treat the leakage points in a reliable manner. Therefore, we believe that the usefulness of PG should be reconsidered. However, there is no contrast agent for the chest cavity, and thus an intravenous contrast agent is used.

In this case series study, we explain the PG method, investigate its rates of detection for air leak points, and examine common complications.

\section{METHODS \\ Eligibility}

PG was approved by the Ethical Committee of Nissan Tamagawa Hospital in the 1980s. ${ }^{3}$ We obtained approval for the present study from our hospital's Ethical Committee in 2018. The Ethical Committee of Nissan Tamagawa Hospital debated the off-label use of Iopamidol 300 for PG and approved its clinical use (approval Tama2018-015). Written informed consent was obtained from all patients after they were apprised of the risks and benefits of the procedure by their surgeon. We used nonionic iodine-based intravenous contrast agents typically used for CT; therefore, patients with multiple drug allergies, asthma, or an estimated glomerular filtration rate (eGFR) $<30 \mathrm{~mL} / \mathrm{min}$ were excluded, in the same way as patients would be excluded from undergoing CT scans. Consent for publication was obtained in the form of opt-out on the website. Those who rejected were excluded from the study.

\section{Patients}

Between March 2015 and October 2018, our institution provided inpatient treatment for 1210 patients with pleural fistula resulting from spontaneous pneumothorax and lung carcinoma. Of these, 275 patients with recurrence of primary spontaneous pneumothorax (PSP), recurrence of secondary spontaneous pneumothorax (SSP), or first treatment SSP were targeted. Only the patients with recurrence of PSP after surgery at our hospital were included in this study.
PG was performed in 127 patients with air leak that persisted during exhalation; 148 patients in whom the air leak was very small and barely detectable or that disappeared were excluded. In addition, 35 patients with complications of postoperative air leakage persisting for $\geq 7$ days were included. All patients provided consent (Figure 1). The 127 patients included 18 with recurrence of PSP, 10 with recurrence of SSP, and 99 with SSP. The causes of recurrence of SSP were Birt-Hogg-Dubé syndrome (BHDS) in 1 patient, lymphangioleiomyomatosis (LAM) in 2 patients, and thoracic endometriotic pneumothorax (TEP) in 7 cases. The causes of SSP were chronic obstructive pulmonary disease (COPD) in 53 patients, interstitial pneumonia in 32 cases, lung cancer in 4 patients, bronchiolitis obliterans in 1 patient, Ehlers-Danlos syndrome in 1 patient, and inflammatory lung diseases, such as nontuberculous mycobacteria, in 8 patients. The patients with LAM, BHDS, and TEP did not undergo PG before the first surgery. Thirty-five patients had postoperative pleural fistula complications, of whom 23 had recurrence of PSP and 12 had SSP. Of the 12 patients with SSP, 6 patients had LAM, 3 patients had BHDS, and 1 patient each had TEP, pulmonary carcinoma with COPD, and pneumonia due to mitochondrial cardiomyopathy.

Table 1 presents the characteristics of 162 patients who underwent PG. The median age of these patients was 57 years (range, 13-90 years). One hundred twenty-six patients ( $78 \%$ ) were men, 87 were affected on the right side, and 82 had an Eastern Cooperative Oncology Group performance status (ECOG PS) of 0, 37 had PS1, 25 had PS2, 12 had PS3, and 6 had PS4. ${ }^{8}$ Sixty-eight patients underwent blood tests, including blood urea nitrogen (BUN) and creatinine ( $\mathrm{Cr}$ ), within 30 days of undergoing PG.

\section{PG Technique}

The PG procedure is as follows (Figure 2). The patient was placed on the radiography table in the supine position. The patient was instructed on desirable conduct during the examination and practiced adopting the posture on the table beforehand. The drainage tube was opened and exposed to the atmosphere, which caused the lung to collapse, and we tested for the presence of adhesions and the degree of lung collapse. At the same time, we tested for the presence of dyspnea and monitored the $\mathrm{SpO}_{2}$ levels and respiratory rate. Subsequently, 20 to $40 \mathrm{~mL}$ of contrast agent (iopamidol; Iopamiron, Bayer HealthCare, Osaka, Japan) was injected via the chest drainage tube. The thoracic drainage tube required a suction of $-20 \mathrm{~cm} \mathrm{H}_{2} \mathrm{O}$ during the procedure and had to be elevated to prevent the injected contrast agent from flowing out. The patients were then shifted to a head-down position so that the apex of the lung could be observed and the right and left anterior oblique views were examined. The radiographic table was returned to a flat position, and the middle of the lung was observed. Then the bottom section of the lung was observed with the patient in the head-up position. If leakage was not detected, the patient adopted a prone position, and the whole lung was observed in the head-down, flat, and head-down positions in a similar manner. When the injected contrast medium reached the leakage point, "bubbling" was observed directly (Video 1; Figure 3). When air leakage was detected, the patient's posture and the incline of the radiography table were recorded. The examination took approximately 30 minutes. If the procedure time is extended beyond this, the contrast medium will get diluted, making observation difficult.

\section{Evaluation of Adverse Events}

Adverse events were graded according to the National Cancer Institute Common Terminology Criteria for Adverse Events, version 4.0. ${ }^{8}$ The toxicities were designated as definitely, probably, possibly, unlikely, or not related to PG. Adverse events unrelated to or unlikely related to PG were not reported. Adverse events were investigated 30 days after the PG procedure. If a patient underwent surgery or any other treatment within this 30-day period, adverse events were investigated only until the next 


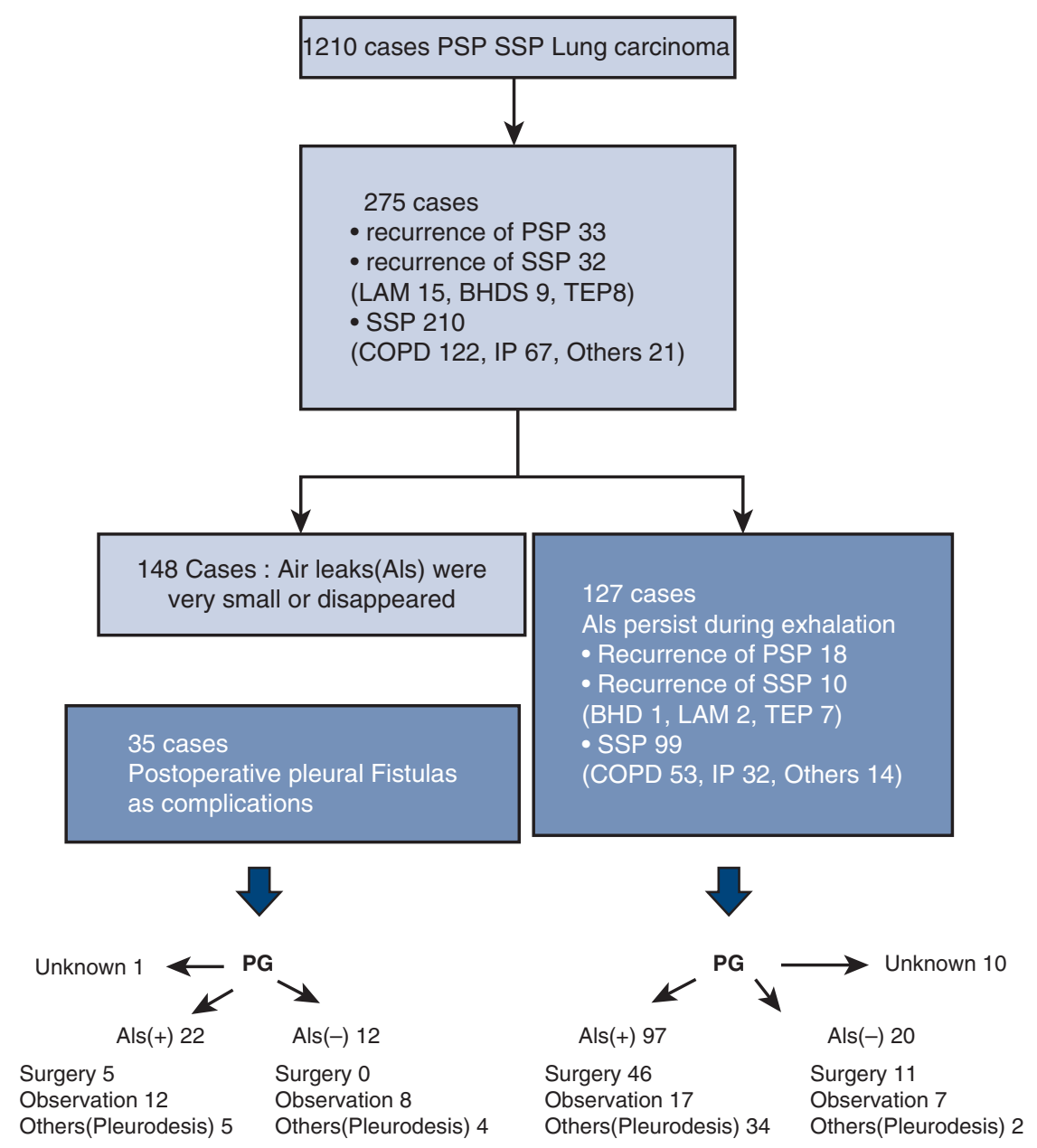

FIGURE 1. Target of 162 patients. SSP of LAM, BHDS, and TEP were included only in recurrent cases. PSP, Primary spontaneous pneumothorax; SSP, secondary spontaneous pneumothorax; LAM, lymphangioleiomyomatosis; BHDS, Birt-Hogg-Dubé syndrome; TEP, thoracic endometoriotic pneumothorax; $C O P D$, chronic obstructive pulmonary disease; $I P$, interstitial pneumonia.; $P G$, pleurography.

treatment was provided. In patients who underwent blood testing, levels of $\mathrm{BUN}, \mathrm{Cr}$, and eGFR were assessed before and after PG, and the values were compared.

\section{Statistics}

Statistical analyses were performed using Microsoft 365 Excel for Windows 10 (Microsoft, Redmond, Wash). Data are expressed as mean or average and standard error.

\section{RESULTS}

Out of 162 cases, 11 patients had no clear medical records regarding the presence or absence of air leaks. The air leak points could be detected in 119 to 130 patients $(73 \%$ to $80 \%)$. Leak points were observed in the apex of the lung in 65 patients $(40 \%)$, in the basal segment in 13 patients $(8 \%)$, and in the middle lobe or lingular segment in 9 patients $(5.6 \%)$. Furthermore, there were 8 patients $(4.9 \%)$ with hilar lesions; $12(7.4 \%)$ with S6 lesions;
TABLE 1. Characteristics of 162 patients who underwent pleurography

\begin{tabular}{lc}
\hline \multicolumn{1}{c}{ Characteristic } & Value \\
\hline Age y, median (range) & $57(13-90)$ \\
Male sex, n (\%) & $126(78)$ \\
Female sex, n (\%) & $36(22)$ \\
ECOG PS, n (\%) & \\
0 & $82(51)$ \\
1 & $37(23)$ \\
2 & $25(15)$ \\
3 & $12(7.4)$ \\
4 & $6(3.7)$ \\
Right, n (\%) & $87(54)$ \\
Left, n (\%) & $74(46)$ \\
Bilateral, n $(\%)$ & $1(0.62)$ \\
\hline
\end{tabular}

ECOG, Eastern Cooperative Oncology Group; $P S$, performance status. 


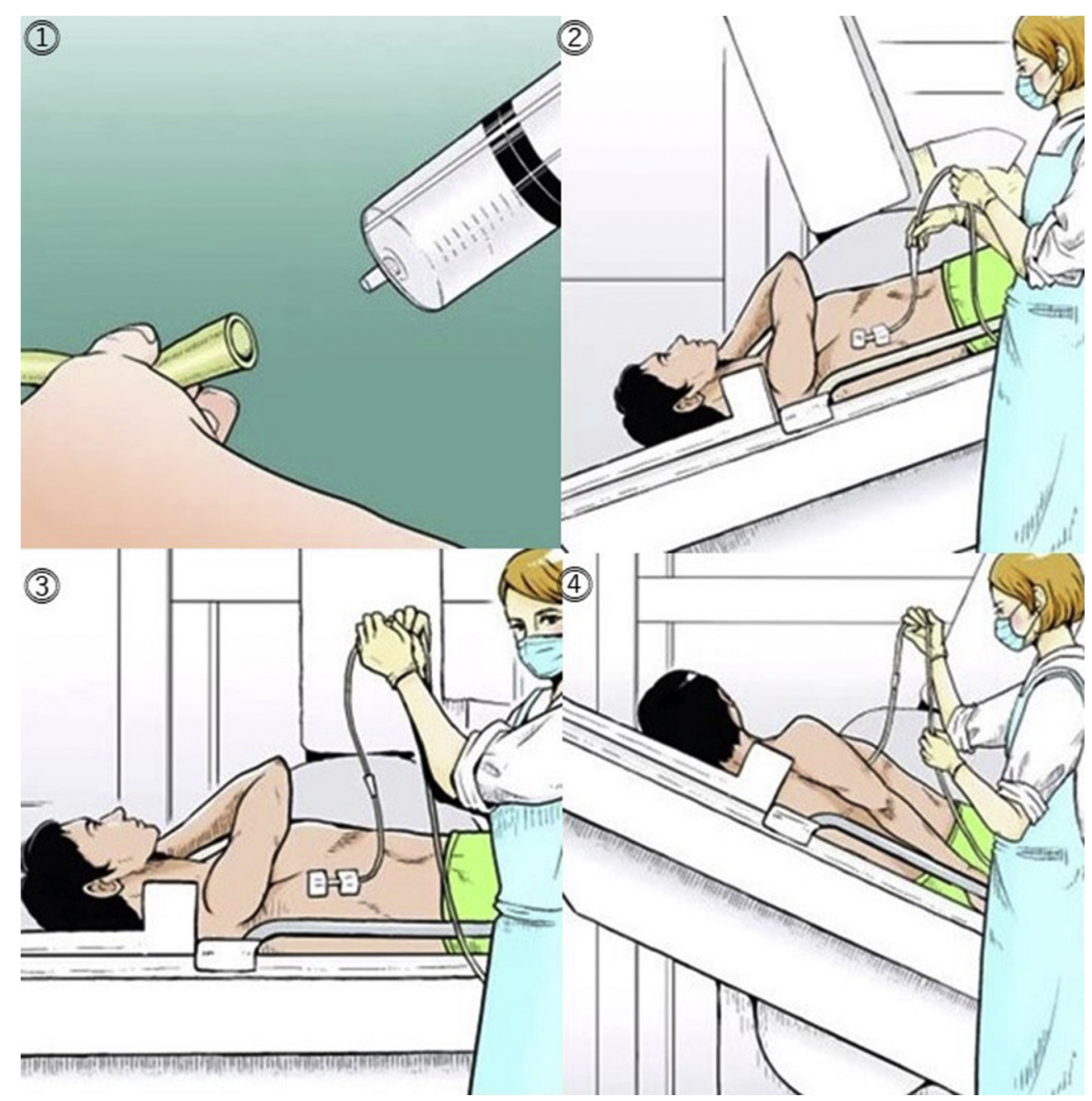

FIGURE 2. Pleurography technique. (1) The drainage tube was opened, which caused the lung to collapse. We verified the presence of adhesions and the degree of collapse of the lung. Subsequently, 20 to $40 \mathrm{~mL}$ of contrast agent (iopamidol) was injected via the chest drainage tube. (2) The patient was placed in a head-down position so that the apex of the lung could be observed. The drainage tube had to be elevated to prevent the injected contrast agent from flowing out. (3) The radiography table was returned to a flat position, and the middle of the lung was observed. (4) We then observed the bottom of the lung in the head-up position, with the patient rolled in different directions and positions.

$8(4.9 \%)$ with S1+2, S2, or S3 lesions; and $4(2.5 \%)$ with upper mediastinal lesions. Air leakage was detected in 68 patients in the supine position, in 38 patients in the prone position, and in 13 patients in the lateral position. Complications were investigated in 162 patients and seen in 10 patients $(6.2 \%)$ within 30 days, with 8 grade 2 cases involving fever, 1 grade 3 case involving infection, and 1 grade 1 case with abdominal distention. The patient with grade 3 infection was diagnosed with empyema at 7 days after the PG, necessitating treatment (Table 2). One patient died on day 30 after PG; however, this patient was diagnosed with terminal COPD, which was judged to be unrelated to the PG. Sixty-eight patients underwent blood tests after PG. The mean BUN, $\mathrm{Cr}$, and eGFR levels were $14 \pm 0.65 \mathrm{mg} / \mathrm{dL}, 0.73 \pm 0.028 \mathrm{mg} / \mathrm{dL}$, and $94 \pm 5.1 \mathrm{~mL} /$ min, respectively, before $\mathrm{PG}$ and $13 \pm 0.59 \mathrm{mg} / \mathrm{dL}$,
$0.71 \pm 0.026 \mathrm{mg} / \mathrm{dL}$, and $94 \pm 4.5 \mathrm{~mL} / \mathrm{min}$ after PG. Therefore, there was no significant difference when Welch's $t$ test was applied (Table 3). Of the 119 patients with detected air leak points, 51 underwent surgery, 29 were placed under observation, and 39 underwent another treatment, such as pleurodesis. Of these, the average operating time for the 51 surgical cases was $93 \pm 8.0$ minutes (range, 20-287 minutes). Of the 32 patients without detected air leak, 11 underwent surgery, 15 were under observation, and 6 underwent other treatments. The average operating time for the 11 surgical patients was $82 \pm 12$ minutes (range, 46168 minutes).

\section{DISCUSSION}

The present case series study demonstrates the safety of PG for identifying air leak points in patients with pleural 


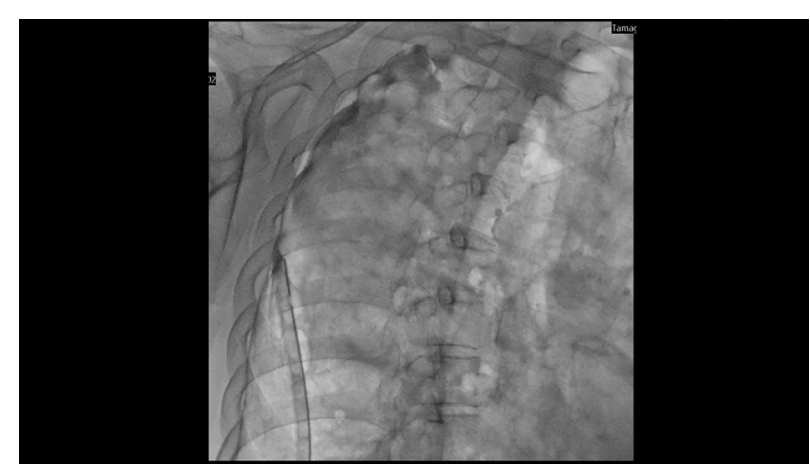

VIDEO 1. Case 1. A 71-year-old man with a postoperative air leak in secondary spontaneous pneumothorax due to chronic obstructive pulmonary disease (COPD). Between 20 and $40 \mathrm{~mL}$ of contrast agent (iopamidol) is injected via the chest drainage tube. The patient is repositioned several times. The contrast medium is carried to the apex of the lungs because there are adhesions in the chest cavity. An air leak point is observed at the apex of the lung. Case 2. A 71-year-old man presented with secondary spontaneous pneumothorax due to COPD. The drainage tube is opened and exposed to the atmosphere, which causes the lung to collapse, and we test for the presence of adhesions and the degree of collapse of the lung. Mild lung collapse is observed. Subsequently, 20 to $40 \mathrm{~mL}$ of contrast agent (iopamidol) is injected via the chest drainage tube. The patient is then moved to a head-down position so that the apex of the lung can be observed. In addition, we can observe adhesion at the apex of the lung. Several bullae are observed at the apex of the lung. The radiography table is returned to a flat position, and we can observe the middle of the lung. Next, we observe the bottom of the lung in the head-up position. If the air leak point is centrally located in the thoracic cavity, it might not be possible to reach the leak point with $40 \mathrm{~mL}$ of the contrast medium. The contrast medium is applied to the surface of the lungs by changing the position, and the search is performed. It is difficult to recognize an air leak near the adhesion of the lung apex. Air leaks and adhesions were also observed in the same area during surgery. Video available at: https://www.jtcvs.org/article/S2666-2507(21)00261-3/fulltext.

fistulas. No contrast medium has been approved for use in the pleural cavity. Hitomi and colleagues ${ }^{1}$ have reported that iodine-based intravenous contrast agents that are injected into the pleural cavity are absorbed in the pleura and discharged from the body in the urine by 24 hours after PG. ${ }^{1}$ The contrast agent dosage was 20 to $40 \mathrm{~mL}$, which is small compared to the dosages used in contrast-enhanced CT. However, careful consideration is required when using these agents in patients with decreased renal function, because they may cause nephropathy. An extensive prospective study is required to obtain additional indications for intravenous contrast medium. In the present study, no patient showed a drop in renal function after PG. In a previous study, adverse reactions to nonionic iodinated contrast media for enhanced CT were reported as follows: any adverse reactions, $4.3 \%$; itching, $1.1 \%$; sneezing, $1 \%$; rashes, $0.3 \%$; and nausea or vomiting, $1.6 \% .{ }^{9}$ In the present study, adverse events due to PG occurred in $6.2 \%$ of patients, with fever the most common adverse reaction. There

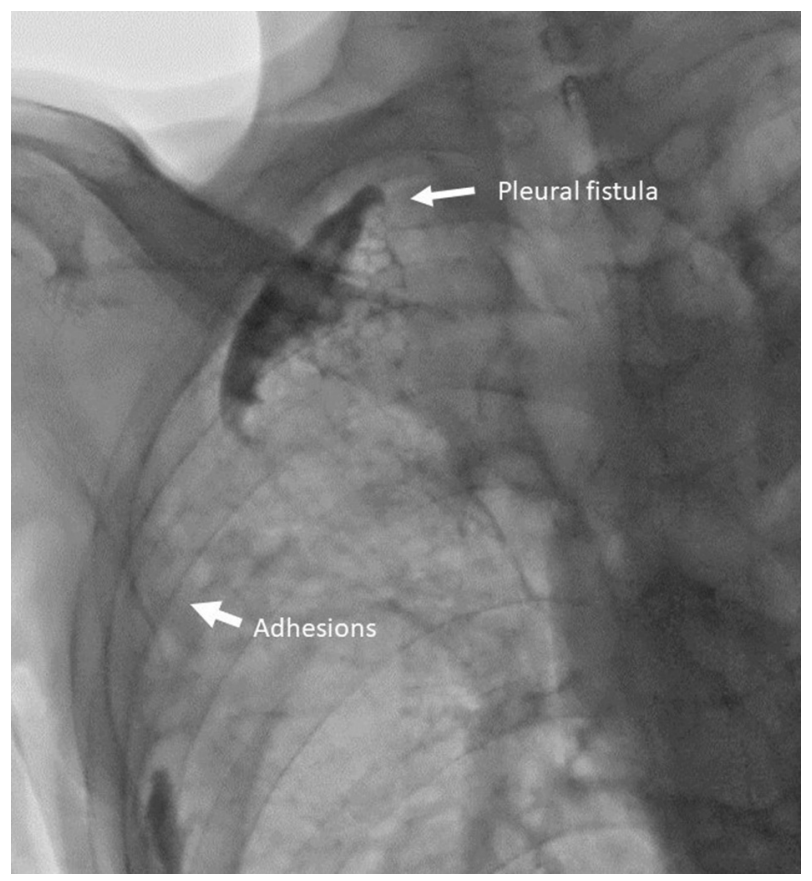

FIGURE 3. Image under fluoroscopy. The drainage tube was opened and exposed to the atmosphere, which caused the lung to collapse. We tested for the presence of adhesions and degree of lung collapse. Subsequently, 20 to $40 \mathrm{~mL}$ of contrast agent (iopamidol) was injected via the chest drainage tube, and the patient was repositioned to observe the pleural cavity. The air leak point is observed in the apex of the lung as air bubbles (arrow). Pleurography shows the location of air leakage in the lungs and the area of adhesions.

were no cases of nausea, vomiting, rash, or itching, suggesting that no patient had an iodine allergy.

Performing PG in patients involving PS4 with respiratory failure or body paralysis is difficult given the patients' inability to adopt or maintain the prone position. Because the head-down and prone positions cause discomfort, fewer observations have been described in the prone position compared with the other positions.

There was no significant difference in operating time between the patients in whom air leak was detected by PG and those in whom air leak was not detected $(P=.28$, Welch's $t$ test). This was not compared with that in the patients who did not undergo PG. Detecting air leak did not reduce the surgical time; however, the case with the shortest operating time was a case in which the air leak point was detected. It was possible to complete the surgery in a 71-year-old

TABLE 2. Adverse events within 30 days after pleurography

\begin{tabular}{lcc}
\hline Complications of pleurography & Grade & Patients, $\mathbf{n}(\%)$ \\
\hline Fever & Grade 2 & $8(5.0)$ \\
Infection & Grade 3 & $1(0.6)$ \\
Abdominal distention & Grade 1 & $1(0.6)$ \\
\hline
\end{tabular}


TABLE 3. BUN, Cr, and eGFR before and after pleurography

\begin{tabular}{lccc}
\hline \multicolumn{1}{c}{ Variable } & Before, mean \pm SE & After, mean \pm SE & $\boldsymbol{P}$ value* \\
\hline BUN, $\mathrm{mg} / \mathrm{dL}$ & $14 \pm 0.65$ & $13 \pm 0.59$ & .31 \\
$\mathrm{Cr}, \mathrm{mg} / \mathrm{dL}$ & $0.73 \pm 0.028$ & $0.71 \pm 0.026$ & .62 \\
$\mathrm{eGFR}, \mathrm{mL} / \mathrm{min}$ & $94 \pm 5.1$ & $94 \pm 4.5$ & .92 \\
\hline
\end{tabular}

$S E$, Standard error; $B U N$, blood urea nitrogen; $C r$, creatinine; $e G F R$, estimated glomerular filtration rate. *Applying Welch's $t$ test.

woman with SSP due to COPD in 20 minutes under local anesthesia via one incision at the appropriate location.

Operating time depends on several factors, such as the existence of severe adhesion, distribution of bullae, and fragility of the lungs. Therefore, a simple comparison may be meaningless or difficult to understand. Operating time was related primarily to the presence or absence of adhesions and did not seem to be affected by the preoperative detection of the air leak. Adhesions can be evaluated by collapsing the lungs using PG. Accurate records of adhesions in the PG were not available in this retrospective study, and no data were provided.

In the event of spontaneous pneumothorax, if the patient's lung is in a collapsed state before drainage, the degree of adhesion can be evaluated by plain chest radiography or CT; however, in postoperative recurrent cases and complications of pleural fistula, it is difficult to evaluate the cause of the adhesion on CT alone. We investigated the findings of intraoperative adhesions in 62 of 162 patients. There were 51 cases of adhesions. The first port is usually placed on the fifth intercostal midaxillary line, but there were 12 cases (19\%) in which the first port was moved to another location because adhesions were found in the thoracic cavity of the fifth intercostal midaxillary area via PG. We speculate that there is scope for further evaluation by PG before surgery.

In some cases of emphysema, collapsing the lung is difficult during surgery, and PG can be used to preoperatively evaluate whether collapse will be difficult. Thoracoscopic surgery is difficult to perform unless the lungs are collapsed, as otherwise a wide surgical field cannot be maintained. When surgery under local anesthesia is indicated, such as in awake video-assisted thorascopic surgery, ${ }^{10} \mathrm{PG}$ should be performed before surgery, because performing the leak test is difficult during thoracoscopic surgery under local anesthesia $^{11}$ (Figure 4).

\section{CONCLUSIONS}

In conclusion, the prevalence of grade $\geq 3$ or higher adverse events associated with PG is $0.6 \%$, which is considered acceptable. Leak points were detected in $73 \%$ of cases by PG. It may be useful as a preoperative examination for awake video-assisted thorascopic surgery, which is difficult to leak test during surgery. By assessing adhesions with PG,

\section{Pleurography(PG) for pleural fistulas: a case series}

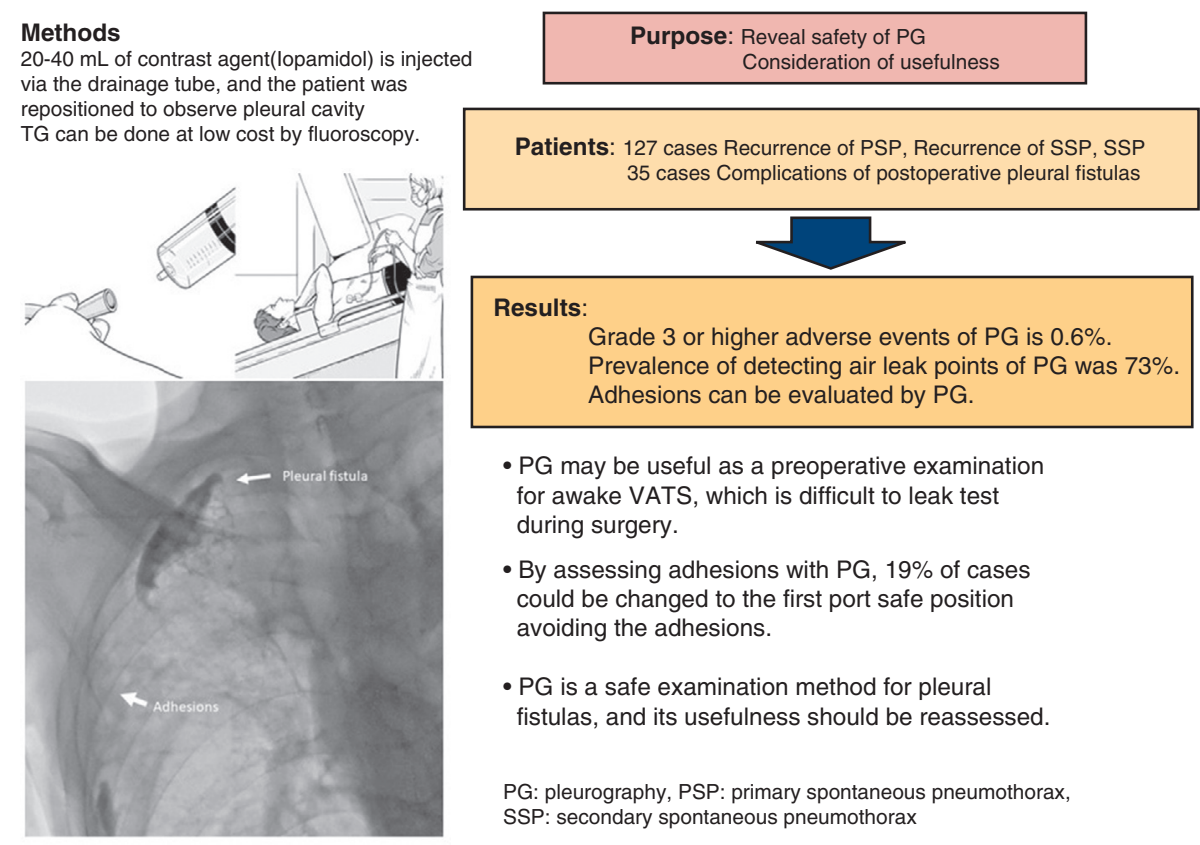

FIGURE 4. Pleurography (PG) can be easily performed with fluoroscopy in any facility. We performed PG in 162 cases; the rate of adverse events of grade $\geq 3$ was $0.6 \%$. Air leak was detected by PG in $73 \%$ of cases. PG may be useful as a preoperative examination for awake video-assisted thorascopic surgery, which is difficult to leak test during surgery. By assessing adhesions with PG, 19\% of cases could be changed to the first port safe position, avoiding the adhesions. PG may provide useful information for surgical management. 
$19 \%$ of cases could be changed to the first port safe position, thereby avoiding the adhesions. Although more prospective research is needed, PG may provide useful information for surgical management. Therefore, we believe that PG should be reconsidered as a useful option for examination.

\section{Conflict of Interest Statement}

The authors reported no conflicts of interest.

The Journal policy requires editors and reviewers to disclose conflicts of interest and to decline handling or reviewing manuscripts for which they may have a conflict of interest. The editors and reviewers of this article have no conflicts of interest.

We thank Dr M Kurihara, other staff, including the radiologic technician at our institution, and Dr K. Seyama from Juntendou University for his valuable comments. We also thank Editage for the English language editing.

\section{References}

1. Hitomi S, Ikeda T, Funatsu T, Sogabe Y, Kai T. Diagnostic value of the contrast thoracography. Kyobu Geka. 1971;24:706-13 [in Japanese].

2. Rudy RL, Bhargava AK, Roenigk WJ. Contrast pleurography. A new technic for the radiographic visualization of the pleura and its various reflections in dogs. Radiology. 1968;91:1034-6.
3. Takeno Y. Thoracoscopic treatment of spontaneous pneumothorax. Ann Thorac Surg. 1993;56:688-90.

4. Matsubara H, Miyauchi Y, Kunimitsu T, Matsuoka H, Ichihara T, Uchida T, et al. Evaluation of thoracography for secondary pneumothorax in elderly people. Kyobu Geka. 2011;64:296-8 [in Japanese].

5. Hallifax RJ, Yousuf A, Jones HE, Corcoran JP, Psallidas I, Rahman NM. Effectiveness of chemical pleurodesis in spontaneous pneumothorax recurrence prevention: a systematic review. Thorax. 2017;72:1121-31.

6. Okereke I, Murthy SC, Alster JM, Blackstone EH, Rice TW. Characterization and importance of air leak after lobectomy. Ann Thorac Surg. 2005; 79:1167-73

7. Nakanishi K, Shimotakahara A, Asato Y, Ishihara T. A new method to detect air leakage in a patient with pneumothorax using saline solution and multidetector-row spiral CT scan. Chest. 2013;144:940-6.

8. Oken MM, Creech RH, Tormey DC, Horton J, Davis TE, McFadden ET, et al Toxicity and response criteria of the Eastern Cooperative Oncology Group. Am J Clin Oncol. 1982;5:649-55.

9. Motosugi U, Ichikawa T, Sano K, Onishi H. Acute adverse reactions to nonionic iodinated contrast media for CT: prospective randomized evaluation of the effects of dehydration, oral rehydration, and patient risk factors. AJR Am J Roentgenol. 2016;207:931-8.

10. Pompeo E, Tacconi F, Mineo D, Mineo TC. The role of awake video-assisted thoracoscopic surgery in spontaneous pneumothorax. J Thorac Cardiovasc Surg. 2007;133:786-90.

11. Watanabe $T$, Noda M, Okazaki $T$, Tsukidate $H$, Sato $K$, Notsuda $H$ Preoperative saline-filled computed tomography thoracography for awake video-assisted thoracic surgery: report of three cases. Surg Today. 2015;45: $1579-82$.

Key Words: thoracography, pleurography, pleural fistula, pneumothorax, fluoroscopy 